\title{
Prevalencia de asma y otras enfermedades alérgicas en niños escolares de Ciudad J uárez, Chihuahua
}

Albino Barraza-Villarreal L.E., M.C, (1) Luz Helena Sanín-Aguirre M.D., D.C., ${ }^{(1,2)}$

Martha María Téllez-Rojo, M.S., M.C.,(1) Marina Lacasaña-N avarro D.C., ${ }^{(1)}$ Isabelle Romieu, M.P.H., SC.D. ${ }^{(3)}$

\section{Barraza-Villarreal A, Sanín-Aguirre LH, Téllez-Rojo MM, Lacasaña-Navarro M, Romiew I. \\ Prevalencia de asma y otras enfermedades alérgicas en niños escolares de Ciudad Juárez, Chihuahua. \\ Salud Publica Mex 2001;43:433-443.} El texto completo en inglés de este artículo está disponible en: http://www.insp.mx/salud/index.html

\section{Resumen}

Objetivo. D eterminar la prevalencia y severidad del asma, de la rinitis y del eczema en escolares. Material y métodos Estudio transversal efectuado entre abril de 1998 y mayo de 1999 en Ciudad Juárez, Chihuahua, México, a una muestra aleatoria de 6174 niños de 53 escuelas. Se aplicó la metodología propuesta por el International Study of Asthma and Allergies in Childhood (ISAAC) (etapas 1 y 2) para determinar la prevalencia y severidad del asma, rinitis y eczema. La información de prevalencia, tanto actual como acumulada para dichos padecimientos, se obtuvo mediante un cuestionario ya estandarizado y contestado por los padres de los niños. El diseño de la muestra se hizo por un muestreo mixto, en el cual se estratificó por nivel de contaminación ambiental. Se estimaron prevalencias actual y acumulada estratificando por grupo de edad, sexo, área e historia familiar de asma. Resultados La prevalencia acumulada de asma por diagnóstico médico y sibilancia (silbidos) fue de $6.8 \%$ (IC 95\% 6.2-7.4) y $20 . \%$ (IC 95\% 19.7-21.8), respectivamente; la prevalencia de sibilancia en los últimos 12 meses fue mayor en el grupo de 6-8 años que en el de $11-14$ años $(9.7 \%)$ contra $5.8 \%(p<0.01)$. La prevalencia de rinitis por diagnóstico médico fue de $5.0 \%$ (IC 95\% 4.5-5.6). La prevalencia de eczema por diagnóstico médico fue de

\author{
Barraza-Villarreal A, Sanín-Aguirre LH, \\ Téllez-Rojo MM, Lacasaña-Navarro M, \\ Romiew I. \\ Prevalence of asthma \\ and other allergic diseases in school \\ children in Ciudad Juarez, Chihuahua. \\ Salud Publica Mex 2001;43:433-443. \\ The English version of this paper \\ is available at: http://www.insp.mx/salud/index.html
}

\begin{abstract}
A bstract
Objective To assess the prevalence and severity of asthma and allergic diseases in schoolchildren residing in Ciudad Juarez, Chihuahua. Material and Methods. A crosssectional study was conducted from A pril 1998 to May 1999, among 6174 children from 53 schools in Ciudad Juarez, Chihuahua. The method used was the one recommended by the International Study of Asthma and Allergies in Childhood (ISAAC) to determine the prevalence and severity of asthma, rhinitis, and eczema. Parents were asked to answer a standardized questionnaire on current and cumulative prevalence of asthma, rhinitis, and eczema.A sample stratified by level of pollution was selected. Results The cumulative prevalence of medically diagno sed asthma and wheezing was $6.8 \%(95 \% \mathrm{Cl} 6.2,7.4)$ and $20 \%(95 \% \mathrm{Cl} 19.7,21.8)$, respectively. The prevalence of wheezing in the last 12 months was higher in the group aged 6-8 years than in those aged $11-14$ years $(9.7 \%$ vs. $5.8 \%, p<0.01)$. The prevalence of medically diagnosed rhinitis was $5.0 \%(95 \%$ C $14.5,5.6)$.The prevalence of medically diagnosed eczema was $4.9 \%$ (4.3, 5.4). The prevalence of eczema symptoms in the last 12 months was $12.7 \%$ in the $6-8$ years group and $13.3 \%$ in the 11-14 year group, respectively. Severe symptoms of asthma were significantly higher in the 6-8 years group and during
\end{abstract}

Estudio realizado con apoyo del $\mathrm{N}$ ational Center for Environmental Health (NCEH/CDC).

(1) Instituto $N$ acional de Salud Pública de México.

(2) Universidad Autónoma de Chihuahua.

(3) Center for Disease Control. 0 rganización Panamericana de la Salud.

Fecha de recibido: 16 de junio de 2000 - Fecha de aprobado: 27 de marzo de 2001

Solicitud de sobretiros: D ra. Marina Lacasaña N avarro. Dirección de Ciencias Ambientales, Instituto N acional de Salud Pública, Avenida Universidad 655, colonia Santa María A huacatitlán, 62508 C uernavaca, Morelos, México. mlacasan@ correo.insp.mx 
4.9\% (IC 95\% 4.3-5.4). La prevalencia de síntomas de eczema en los últimos 12 meses fue de $12.7 \%$ en el grupo de 6 8 años y de $13.3 \%$ en el de 11-14 años. Los sínto mas severos de asma fueron significativamente más prevalentes en el grupo de 6-8 años y en los meses de otoño. Conclusiones La prevalencia de asma, tanto por diagnóstico médico como por síntomas, resulta relativamente baja en relación con otros estudios realizados con la misma meto dolo gía, pero las prevalencias de rinitis y eczema fueron altas. El texto completo en inglés de este artículo está disponible en:http:/ /www.insp.mx/salud/index.html

Palabras clave: prevalencia; asma; rinitis; dermatitis atópica; niño; México the autumn months. Conclusions. The prevalence of medically diagno sed and sympto matic asthma was relatively low in comparison with findings from others studies that use similar methods, but the prevalence rates of rhinitis and eczema were higher.
E l asma es la enfermedad más común que se presenta en los niños de Estados Unidos de América (EUA), afectando aproximadamente a 2.7 millones. ${ }^{1}$ El análisis de datos de The National Health Interview Survey reveló que, entre 1981 y 1988, la prevalencia del asma informada por los padres de familia tuvo un incremento de $42 \%$ en todos los niños menores de 18 años. ${ }^{1}$ En la década pasada, las hospitalizaciones por asma en los Estados Unidos tuvieron un incremento de $46 \%$ entre los niños menores de cinco años de edad, y las tasas de mortalidad correspondientes tuvieron un incremento de $80 \%$ entre los niños de 5-14 años de edad. $^{2}$

Diversos estudios en el ámbito mundial han sido realizados con la finalidad de hacer comparaciones internacionales sistemáticas sobre prevalencias de asma y otras enfermedades alérgicas. Investigaciones realizadas por el Comité del International Study of Asthma and Allergies in Childhood (ISAAC) en diferentes centros de 56 países han encontrado diferencias de entre 20 y $60 \%$ en las prevalencias de síntomas de asma entre los niños de 13 y 14 años de edad, y han servido como base para futuras investigaciones que permitan conocer los posibles patrones de estas enfermedades en el ámbito internacional. ${ }^{3}$

Estudios realizados en Finlandia, Suecia, Australia, Nueva Zelanda y Gran Bretaña, y en países de América Latina como Brasil, Puerto Rico y México han hallado que la prevalencia del asma se ha ido incrementando en la última década; algunos otros revelan que la mortalidad por asma, medida a través de las defunciones que ocurren en los pacientes hospitalizados, ha tenido un incremento, particularmente entre los niños.

La rinitis alérgica constituye la enfermedad crónica más común de las vías respiratorias altas; esta última se presenta aproximadamente en $10 \%$ de los niños de EUA, y en más de $20 \%$ de adolescentes y adultos jóvenes; la prevalencia de dermatitis atópica en EUA y Gran Bretaña se calcula entre 1.9 y $5 \%{ }^{4}$

Este tipo de enfermedades tiene, además, un amplio impacto sobre la actividad de los niños y adolescentes. Diferentes estudios señalan que las personas con algún tipo de padecimiento crónico tienen que permanecer, en promedio, 3.4 días en cama o faltar hasta 4.4 días a la escuela. ${ }^{5}$

En todo el mundo se han realizado múltiples estudios, predominantemente de tipo transversal, con la finalidad de determinar la prevalencia de estas enfermedades y observar su tendencia.

Se considera que existen más de 10 millones de personas que presentan asma en los EUA, y diversos estudios en algunas ciudades arrojan información sobre prevalencias que oscilan entre 6.4 a $10.8 \%$ en niños de 7 a 8 años de edad. ${ }^{6}$ Por otro lado, desde el punto de vista epidemiológico, se ha visto una ligera diferencia con relación al sexo y a la raza: afecta en mayor proporción al sexo masculino y a los de raza negra; no existen cambios importantes en lo referente a edad infantil.

Los datos comunicados de prevalencia de asma, según estudios hechos en diferentes ciudades de México, arrojan información que va desde 2.7 hasta $21.8 \%$, variando notablemente de una región a otra. ${ }^{4,7-10} \mathrm{La}$ prevalencia arrojada por el único estudio realizado en este país, utilizando la metodología de ISAAC, fue de $5.7 \mathrm{y}$ de $5.9 \%$ para los dos grupos de edad estudiados. ${ }^{11}$

Moyes y colaboradores, utilizando la metodología ISAAC en 1995, efectuaron un estudio con el propósito de conocer la prevalencia de síntomas respiratorios en niños, examinando la asociación con factores ambientales. $^{12}$

Con el fin de explorar el comportamiento de estos padecimientos se realizó el estudio en el área metro- 
politana de Ciudad Juárez, Chihuahua, una de las principales ciudades fronterizas de nuestro país y uno de los municipios más importantes del estado de Chihuahua. Está situada al norte de la República Mexicana, a una longitud de $106^{\circ} 29^{\prime} 01^{\prime \prime}$ y una latitud de $36^{\circ} 44^{\prime}$ $18^{\prime \prime}$, tiene una altura de 1277 metros sobre el nivel del mar y una extensión territorial de $4853.84 \mathrm{~km}^{2}$. Según cálculos realizados por la Dirección de Estadísticas de Ciudad Juárez (DECJ) sobre la base del ritmo de crecimiento, y según datos del Instituto Nacional de Estadística, Geografía e Informática (INEGI) del XI Censo General de Población y Vivienda de 1990,13 Ciudad Juárez contaba con un total de 1000363 habitantes para marzo de 1995. Tiene un clima extremoso y alcanza temperaturas en primavera-verano de $46^{\circ} \mathrm{C}, \mathrm{y}$ temperaturas mínimas en invierno de hasta $-5{ }^{\circ} \mathrm{C}$.

Según cálculos del Departamento de Planeación, Investigación y Desarrollo del Departamento de Tráfico y Transportación de la Ciudad de El Paso Texas, en el año de 1995 circularon de esa ciudad al municipio de Juárez un total de 2259828 vehículos no comerciales y un total de 117945 vehículos comerciales, los cuales, sumados al total de los que normalmente circulan en esta ciudad y al incremento en el número de industrias maquiladoras que se ha venido suscitando, desencadena un problema de contaminación que afecta principalmente la salud de sus habitantes y en especial a las personas más susceptibles a estos fenómenos: los niños y los ancianos.

El presente estudio se llevó a cabo utilizando la propuesta del ISAAC, cuya metodología ISAAC propone la realización de los estudios en tres etapas: la primera contempla el investigar sólo la prevalencia y severidad del asma, rinitis y eczema; la segunda, basada en los hallazgos de la primera, incorpora los factores de riesgo asociados y la aplicación de pruebas de función pulmonar; la tercera recomienda que después de haber transcurrido cinco años de haber realizado la primera etapa, se realice nuevamente el estudio para ver su evolución y posibles tendencias. La presente investigación informa sobre los resultados de la primera etapa de dicha metodología en Ciudad Juárez, Chihuahua. ${ }^{6,14-18}$

\section{Material y métodos}

La investigación se planeó de acuerdo con las áreas potenciales de contaminación de Ciudad Juárez, Chihuahua, la cual se dividió en tres grandes estratos sugeridos por un experto. El estrato 1 correspondió a la zona centro de la ciudad y, con base en los datos de material particulado y en la gran cantidad de vehículos que circulan por dicha área, se consideró el estrato de mayor contaminación. El estrato 2 abarcó la zona más grande de la ciudad y fue considerado medianamente contaminado. El estrato 3 correspondió al área periférica de la ciudad y se consideró el menos contaminado.

Se utilizó el programa STATCALC del programa EPI5 para calcular el tamaño de muestra necesario, para obtener un poder suficiente y poder detectar diferencias mínimas de $3 \%$ en la prevalencia entre ciudades y diferencias de $5 \%$ en la prevalencia de severidad de los casos estudiados, de acuerdo con las especificaciones del ISAAC y dado el carácter multicéntrico del estudio. Así, se eligió una muestra probabilística de 7200 niños en edad escolar: 3000 niños en el grupo de 6 a 8 años y 3000 en el grupo de 11 a 14 años, más un $20 \%$ por posibles pérdidas, que coincidió con lo propuesto por ISAAC. ${ }^{19}$

Con la colaboración de la Secretaría de Educación Pública (SEP) del estado de Chihuahua, se obtuvo información de las escuelas primarias de Ciudad Juárez, lo cual permitió elaborar el marco muestral. Se hizo una asignación proporcional de la muestra al tamaño del estrato y se consideraron las escuelas como conglomerados. Al final, la muestra quedó constituida por 53 escuelas en las cuales se muestreó al total de alumnos de los grupos de primero y sexto grado. La etapa de campo se inició en abril de 1998 y terminó en mayo de 1999.

Se elaboró y adaptó el cuestionario de ISAAC incluyendo datos sociodemográficos generales y la historia clínica. Para determinar la predisposición hereditaria se preguntó sobre el antecedente familiar de asma en el padre, la madre o alguno de los hermanos del niño participante, y se hizo énfasis en los problemas de tipo respiratorio y en la presencia de síntomas y diagnóstico médico. Para establecer la severidad del padecimiento se preguntó acerca de la frecuencia con que se presentaba la sintomatología, presencia de los síntomas de sibilancia posterior al ejercicio, si dicha sintomatología impedía la manera de hablar del niño, uso de medicamentos y la necesidad de acudir al servicio de urgencias, frecuencia con que ha faltado a la escuela, así como la interferencia del padecimiento con la vida cotidiana. Para el caso de rinitis y eczema se interrogó igualmente sobre diagnóstico médico de estos padecimientos, sobre síntomas específicos, frecuencia y gravedad.

Las definiciones operacionales utilizadas para asma fueron: diagnóstico médico de asma, diagnóstico médico de bronquitis asmática, diagnóstico médico de asma y bronquitis asmática; por síntomas, las definiciones fueron: sibilancia alguna vez, sibilancia en los últimos 12 meses, sibilancia posterior a ejercicio, tos 
persistente (presencia de tos por tres o más semanas), tos nocturna y tos posterior a ejercicio; las definiciones para establecer la severidad del padecimiento fueron: sibilancia sin gripa o catarro, interferencia con el sueño, el lenguaje y las actividades escolares, así como la frecuencia y la necesidad de atención médica o uso de fármacos.

Para rinitis, la definición operacional fue, además del diagnóstico médico, la presencia de síntomas nasales sin gripa, síntomas nasales más oculares y, como indicador de severidad, el grado de interferencia con la vida cotidiana.

En el caso del eczema, además del diagnóstico, la definición operacional incluyó la presencia de "ronchas" alguna vez y "ronchas" en los últimos 12 meses, y como indicadores de severidad, la frecuencia de comezón nocturna y su grado de interferencia con el sueño.

El análisis se llevó a cabo utilizando el paquete estadístico STATA 5.0 y se concluyó en noviembre de 1999. Se realizó el análisis descriptivo de los datos y se determinaron la prevalencia actual y la acumulada, así como la prevalencia de la severidad de los padecimientos tanto por diagnóstico médico como por síntomas de asma, rinitis y eczema. Finalmente, se hizo análisis estratificado por grupo de edad, sexo, área o estrato e historia familiar de asma.

\section{Resultados}

De un total de 6749 niños elegibles, participaron en el estudio 6200 , teniendo una tasa de respuesta de $92 \%$. No fueron incluidos 26 participantes en el análisis por no pertenecer a los grupos de edad establecidos en el diseño. La muestra quedó constituida por 3082 niños y por 3092 niñas; de todos, 3390 tenían de 6 a 8 años de edad y 2784 de 11 a 14 años. Los de 6 a 8 años correspondieron al grupo de primer grado y los de 11 a 14 años al grupo de sexto grado.

El nivel socioeconómico bajo constituye un factor de riesgo que se ha relacionado con una mayor mortalidad por asma. En el estudio se discriminó el pertenecer a escuela pública o privada, así como el nivel de educación materna como una aproximación al nivel socioeconómico. De la muestra, 92.5\% de las escuelas pertenecían al sistema público y $7.5 \%$ al sistema privado; en cuanto a la escolaridad materna se observó que $2.2 \%$ de las madres no tuvieron ninguna escolaridad, 49.1\% contaba únicamente con educación básica, $32.8 \%$ con educación media y sólo $8.2 \%$ de la muestra tuvo nivel profesional.

Los resultados de la prevalencia por diagnóstico médico y por síntomas para asma, rinitis y eczema por grupo de edad y para el total de participantes se encuentran resumidos en los cuadros I, II y III.

Para determinar la prevalencia de asma se tomó en cuenta el diagnóstico médico tanto para la definición de asma como para bronquitis asmática, ya que ambos términos se utilizan en la práctica médica para clasificar a un niño como asmático. En el análisis se unieron estas dos definiciones y se obtuvo una prevalencia para diagnóstico médico de asma y bronquitis asmática de 6.8\% para el grupo total. En referencia a los grupos de edad, para los de primer año (6 a 8 años) la prevalencia encontrada fue de $6.7 \%$, y para el grupo de sexto año (11 a 14 años) fue de $7.4 \%$, que posiblemente se acerque más a la prevalencia por diagnóstico médico real (cuadro I).

Por síntomas, las prevalencias más altas se presentaron en los de menor edad ( 6 a 8 años) encontrando una diferencia estadísticamente significativa en los siguientes síntomas: sibilancia alguna vez en la vida, sibilancia en los últimos 12 meses, tos persistente en los últimos 12 meses, tos nocturna y tos posterior a ejercicio. La prevalencia de sibilancia posterior a esfuerzo físico, la cual representa posiblemente al grupo de los hiperreactores, presentó igualmente una ligera diferencia en los grupos, y fue mayor en los de menor edad (cuadro I). Al analizar los datos por síntomas de severidad, se observó una mayor prevalencia entre los niños de 6 a 8 años que es significativa en las siguientes variables: niños que se despiertan más de una noche por semana, $(6.5 \%$ contra $3.7 \% p<0.01)$, dificultad para hablar por sibilancia (3.5\% contra $2.8 \% p<0.01)$, uso de medicamento para el control del asma $(7.5 \%$ contra $4.4 \% p<0.01)$, ausentismo escolar por tres o más días $(3.2 \%$ contra $1.9 \% p<0.01)$ y atención en el servicio de urgencias $(7.7 \%$ contra $5.7 \% p<0.01)$ (cuadro I).

$\mathrm{Al}$ estratificar por sexo, tanto la prevalencia de asma por diagnóstico médico como por síntomas, no mostraron diferencias significativas. De los niños de la muestra cuyos padres informaron acerca del inicio de síntomas de sibilancia, $8.2 \%$ presentó síntomas de sibilancia entre la etapa de recién nacido y el año de edad, $5.3 \%$ presentó sintomatología entre los 2 y 3 años de edad y $2.7 \%$ entre los 4 a 5 años. En este último periodo $16.2 \%$ de los niños había presentado ya alguna manifestación de la enfermedad. En relación con la prevalencia de síntomas de tos y sibilancia por mes de presentación se observa un incremento en la sintomatología a finales de otoño e invierno, meses de bajas temperaturas y vientos frecuentes.

Entre los factores desencadenantes de sibilancias, la mayor prevalencia fue de $5.23 \%$ para el invierno, donde prevalece el clima frío, seguida por la de infección $(2.3 \%)$ y de ejercicio $(1.5 \%)$; las demás causas 


\section{Cuadro I \\ Prevalencia de asma por diagnóstico médico y por síntomas. Ciudad Juárez, Chihuahua, México, 1998-1999}

\begin{tabular}{|c|c|c|c|c|c|c|c|c|c|}
\hline \multirow[b]{2}{*}{ Variable } & \multicolumn{3}{|c|}{ Todas las edades } & \multicolumn{3}{|c|}{6 a 8 años } & \multicolumn{3}{|c|}{11 a 14 años } \\
\hline & $n$ & $\%$ & IC95\% & $\mathrm{n}$ & $\%$ & IC95\% & $n$ & $\%$ & IC95\% \\
\hline Asma* & 264 & 4.3 & $3.8-4.8$ & 143 & 4.2 & $3.5-4.9$ & 121 & 4.4 & $3.6-5.2$ \\
\hline Bronquitis asmática* & 302 & 4.9 & $4.4-5.5$ & 169 & 5.0 & $4.3-5.8$ & 133 & 4.8 & $4.1-5.7$ \\
\hline Asma y bronquitis asmática* & 419 & 6.8 & $6.2-7.4$ & 227 & 6.7 & $5.9-7.6$ & 192 & 7.4 & $6.4-8.4$ \\
\hline
\end{tabular}

Síntomas

\begin{tabular}{lrrrrrrrrr} 
Sibilancia alguna vez* $^{*}$ & 1271 & 20.7 & $19.7-21.8$ & 759 & 22.4 & $21-24$ & $17-20^{\S}$ \\
\hline Sibilancia actual* & 491 & 7.9 & $7.3-8.6$ & 329 & 9.7 & $8.7-11.0$ & 16.4 & 5.8 & $5.0-6.8^{\S}$ \\
\hline Sibilancia posterior a ejercicio* $^{\S}$ & 291 & 4.7 & $4.2-5.3$ & 175 & 5.2 & $4.4-5.9$ & 116 & 4.2 & $3.5-5.0^{\S}$ \\
\hline Tos persistente $^{\ddagger}$ & 1631 & 26.4 & $25.6-27.8$ & 1069 & 31.53 & $29.9-33.1$ & 562 & 20.2 & $19-22^{\S}$ \\
\hline Tos nocturna $^{\ddagger}$ & 1167 & 18.9 & $17.9-19.9$ & 783 & 23.1 & $22-25$ & 384 & 13.8 & $13.0-15.1^{\S}$ \\
\hline Tos posterior a ejercicio $^{\ddagger}$ & 926 & 15.0 & $14.1-15.9$ & 616 & 18.2 & $16.9-19.5$ & 310 & 11.2 & $10.0-12.4^{\S}$
\end{tabular}

Síntomas de severidad

$\begin{array}{lllllllll}\text { Sibilancia sin gripa o catarro }{ }^{\ddagger} & 153 & 2.5 & 2.1-2.9 & 101 & 2.9 & 2.4-3.6 & 1.9 & 1.4-2.5^{\S}\end{array}$

Despierta por sibilanciał

\begin{tabular}{lrrrrrrrrr}
$<1$ noche por semana & 321 & 5.2 & $4.68-5.81$ & 219 & 6.5 & $5.6-7.4$ & 102 & 3.7 & $3.0-4.5^{\S}$ \\
\hline$>1$ noche por semana & 103 & 1.7 & $1.37-2.0$ & 58 & 1.7 & $1.3-2.2$ & 45 & 1.6 & $1.2-2.2^{\S}$ \\
\hline Más de 3 episodios de sibilancia ${ }^{\ddagger}$ & 99 & 1.6 & $1.3-1.9$ & 55 & 1.6 & $1.2-2.1$ & 44 & 1.6 & $1.2-2.2^{\S}$ \\
\hline Dificultad para hablar por sibilancia & 194 & 3.2 & $2.7-3.6$ & 118 & 3.5 & $3.0-4.2$ & $2.2-3.4^{\S}$
\end{tabular}

Faltó a la escuela tres o más veces

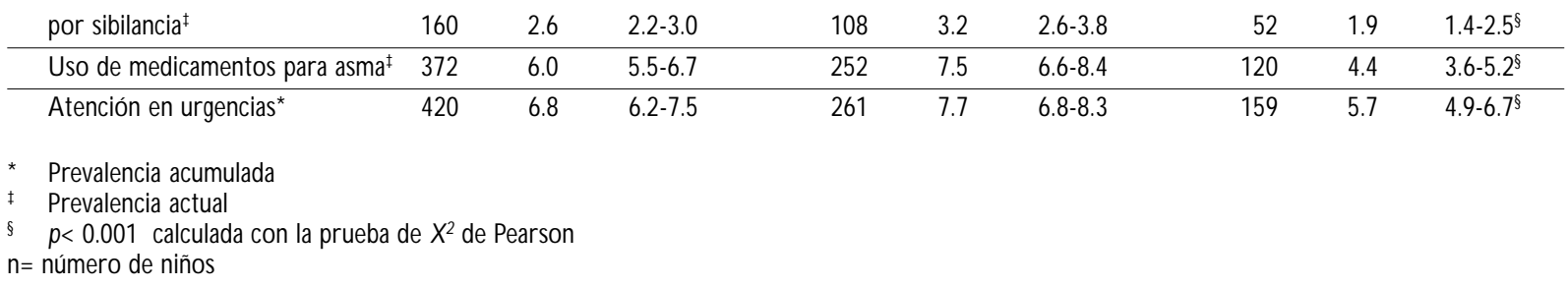

evaluadas (polvo al barrer, humedad en el ambiente, humo, calor y contacto en la casa con animales) mostraron prevalencias muy bajas. Otro de los factores considerado como desencadenante de la crisis de asma es el humo de tabaco. En el presente estudio 33\% de los padres dijeron fumar en presencia de los hijos, y de éstos, $11 \%$ fuma entre 3 y 10 cigarrillos al día.

Las prevalencias de rinitis por diagnóstico médico y por síntomas se encuentran resumidas en el cuadro II. La prevalencia de rinitis por diagnóstico médico fue significativamente más alta en el grupo de 11 a 14 años que en el de 6 a 8. Con relación a los síntomas típicos de rinitis y síntomas de severidad, no se encontraron diferencias importantes por grupo de edad. Por sexo, la prevalencia fue mayor en el masculino, diferencia no significativa para las diferentes patologías estudiadas.

La prevalencia de rinitis fue determinada también de acuerdo con el mes de presentación, mostrando, al igual que la prevalencia de tos y sibilancia, el mismo patrón estacional.

La prevalencia de eczema por diagnóstico médico y por síntomas se presenta en el cuadro III. No se encontraron diferencias importantes para diagnóstico médico entre los grupos comparados. Con relación a los síntomas de la enfermedad, la prevalencia acumulada de eczema fue significativamente mayor para el grupo de 11 a 14 años. Con relación a los síntomas de severidad, no se encontraron diferencias entre ambos grupos y se observaron prevalencias muy bajas. 


\section{Cuadro II \\ Prevalencia de rinitis por diagnóstico médico y por síntomas. \\ Ciudad Juárez, Chinuahua, México, 1998-1999}

\begin{tabular}{|c|c|c|c|c|c|c|c|c|c|}
\hline \multirow[b]{2}{*}{ Variable } & \multicolumn{3}{|c|}{ Todas las edades } & \multicolumn{3}{|c|}{6 a 8 años } & \multicolumn{3}{|c|}{11 a 14 años } \\
\hline & $\mathrm{n}$ & $\%$ & IC $95 \%$ & $\mathrm{n}$ & $\%$ & IC $95 \%$ & $\mathrm{n}$ & $\%$ & IC $95 \%$ \\
\hline Rinitis* & 308 & 5.0 & $4.5-5.6$ & 158 & 4.7 & $4.0-5.5$ & 150 & 5.4 & $4.6-6.3$ \\
\hline \multicolumn{10}{|l|}{ Síntomas de rinitis } \\
\hline Síntomas nasales sin gripa ${ }^{\ddagger}$ & 1772 & 28.8 & $28.0-30.0$ & 974 & 28.8 & $27.3-30.4$ & 798 & 29.0 & $27.0-31.0^{\S}$ \\
\hline Síntomas nasales más oculares ${ }^{\ddagger}$ & 1016 & 16.6 & $15.6-17.5$ & 572 & 17.0 & $15.7-18.2$ & 444 & 16.1 & $15.0-17.4^{\S}$ \\
\hline \multirow{2}{*}{\multicolumn{10}{|c|}{$\begin{array}{l}\text { Síntomas de severidad } \\
\text { Rinitis }\end{array}$}} \\
\hline & & & & & & & & & \\
\hline \multicolumn{10}{|l|}{ Interferencia con la vida cotidiana $a^{\ddagger}$} \\
\hline Poco & 1454 & 23.8 & $23.0-25.0$ & 823 & 24.5 & $23.0-26.0$ & 631 & 23 & $21.3-25.0^{5}$ \\
\hline Mucho & 82 & 1.3 & $1.1-1.7$ & 47 & 1.4 & $1.0-1.9$ & 35 & 1.3 & $0.90-1.8)^{\S}$ \\
\hline \multicolumn{10}{|c|}{$\begin{array}{l}\text { * Prevalencia acumulada } \\
\text { ₹ Prevalencia actual } \\
\S p<0.001 \text { calculada con la prueba de } \chi^{2} \text { de Pearson } \\
n=\text { número de niños }\end{array}$} \\
\hline
\end{tabular}

Cuadro III

Prevalencia de eczema por diagnóstico médico y por síntomas.

Ciudad Juárez, Chihuahua, México, 1998-1999

\begin{tabular}{|c|c|c|c|c|c|c|c|c|c|}
\hline \multirow[b]{2}{*}{ Variable } & \multicolumn{3}{|c|}{ Todas las edades } & \multicolumn{3}{|c|}{6 a 8 años } & \multicolumn{3}{|c|}{11 a 14 años } \\
\hline & $n$ & $\%$ & IC $95 \%$ & $n$ & $\%$ & IC 95\% & $n$ & $\%$ & IC 95\% \\
\hline Eczema* & 298 & 4.9 & 4.3-5.4 & 166 & 4.9 & $4.2-5.7$ & 132 & 4.8 & $4.0-5.7$ \\
\hline \multicolumn{10}{|l|}{ Síntomas de eczema } \\
\hline Ronchas alguna vez* & 795 & 13 & $12.0-14.0$ & 429 & 12.7 & $12.0-14.0$ & 366 & 13.3 & $12.0-15.0$ \\
\hline Ronchas últimos 12 meses $^{\ddagger}$ & 525 & 8.5 & $7.8-9.2$ & 290 & 8.6 & $7.6-9.6$ & 235 & 8.5 & $7.5-9.6$ \\
\hline \multicolumn{10}{|l|}{ Síntomas de severidad } \\
\hline \multicolumn{10}{|l|}{ Eczema } \\
\hline \multicolumn{10}{|c|}{ Despierta en la noche por comezón } \\
\hline$<$ de 1 vez por semana $a^{\ddagger}$ & 43 & 0.69 & $0.5-0.9$ & 24 & 0.71 & $0.46-1.1$ & 19 & 0.69 & $0.41-1.1$ \\
\hline$>$ de 1 vez por semana $a^{\ddagger}$ & 37 & 0.60 & $0.4-0.8$ & 15 & 0.44 & $0.25-0.73$ & 22 & 0.79 & $0.50-1.2$ \\
\hline $\begin{array}{l}\text { * Prevalencia acumulada } \\
\begin{array}{l}\text { Prevalencia actual } \\
\S p<0.001 \text { calculada con la prue } \\
n=\text { número de niños }\end{array}\end{array}$ & d & & & & & & & & \\
\hline
\end{tabular}

Al estratificar por sexo, la prevalencia por diagnóstico médico y por síntomas fue mayor en el femenino. Sin embargo, la diferencia observada no fue estadísticamente significativa.

La herencia es otro factor considerado como predisponente en el desarrollo de estos tres padeci- mientos. En el cuadro IV se resumen los resultados del análisis por antecedentes familiares de asma. Como se puede observar, la prevalencia de estas patologías, tanto por diagnóstico médico como por síntomas, es mucho mayor en los niños que tuvieron antecedentes familiares de asma, en comparación con aquellos que 
no tuvieron esta condición. La diferencia entre estos dos grupos fue en todos los casos estadísticamente significativa.

En el análisis por tipo de escuela (cuadro V), la prevalencia por diagnóstico médico de asma, rinitis y eczema fue mayor entre los niños que acudieron a escuela privada, siendo esta diferencia estadísticamente significativa para todos los padecimientos en cuestión. Por síntomas, la prevalencia siempre fue mayor en los niños de escuela pública y que son los de menor nivel socioeconómico, siendo esta diferencia estadísticamente significativa en tos persistente y tos nocturna. Para eczema se encontró igualmente una mayor prevalencia de síntomas entre los niños de escuelas públicas siendo estadísticamente significativa (13.2\% contra $11.0 \% p<0.01)$ y $(8.6 \%$ contra $7.9 \% p<0.01)$ respectivamente (cuadro V).
Para el caso de síntomas de rinitis, las prevalencias encontradas fueron de $28.3 \%$ contra $37.1 \%(p<0.01)$ y $16.6 \%$ contra $16.2 \%(p<0.01)$, respectivamente (cuadro V).

En cuanto al análisis por estratos, se encontró que las prevalencias más altas fueron para diagnóstico médico de asma y bronquitis asmática $(7.6 \%)$ y para rinitis (5.2\%); para el caso del eczema la prevalencia más alta fue para el estrato $3(5.3 \%)$. En relación con la sintomatología llama la atención que las prevalencias de síntomas de asma fueron más altas en el estrato 3 $(27.0 \%, 36.0 \% 20.4 \%$ y $7.7 \%)$, que corresponden a tos nocturna, tos persistente, sibilancia alguna vez y sibilancia en los últimos 12 meses, respectivamente. Para síntomas de eczema: presencia alguna vez de manchas resecas en la piel, con comezón, que aparezcan y desaparezcan por temporadas de al menos seis meses; la

\section{Cuadro IV}

Prevalencia de asma, Rinitis y eCzema por antecedentes familiares. Ciudad Juárez, Chihuahua, México, 1998-1999

\begin{tabular}{|c|c|c|c|c|c|c|}
\hline \multirow[b]{2}{*}{ Variable } & \multicolumn{3}{|c|}{ Con antecedentes familiares } & \multicolumn{3}{|c|}{ Sin antecedentes familiares } \\
\hline & $\mathrm{n}$ & $\%$ & IC95\% & $\mathrm{n}$ & $\%$ & IC95\% \\
\hline \multicolumn{7}{|l|}{ Por diagnóstico médico } \\
\hline Asma* & 84 & 16.7 & $13.6-20.3$ & 180 & 3.2 & $2.8-3.7$ \\
\hline Bronquitis asmática* & 75 & 15.0 & $12.0-18.3$ & 227 & 4.0 & $3.5-4.6$ \\
\hline Asma y bronquitis asmática* & 109 & 21.6 & $18.1-26.0$ & 310 & 5.5 & $4.9-6.1$ \\
\hline Rinitis* & 39 & 7.8 & $5.6-10.4$ & 269 & 4.8 & $4.2-5.4$ \\
\hline Eczema* & 38 & 7.6 & $5.4-10.3$ & 260 & 4.6 & $4.1-5.2$ \\
\hline
\end{tabular}

Por síntomas, asma

\begin{tabular}{lrrrrrc} 
Tos nocturna $^{\ddagger}$ & 124 & 24.7 & $21.0-29.0$ & 1043 & 18.4 & $17.4-19.4$ \\
\hline Tos persistente $^{\ddagger}$ & 174 & 34.6 & $30.4-39.0$ & 1457 & 26.0 & $25.0-27.0$ \\
\hline Sibilancia alguna vez* & 175 & 35.0 & $31.0-40.0$ & 1096 & 19.4 & $18.4-20.5$ \\
\hline Sibilancia actual $^{\ddagger}$ & 84 & 16.1 & $13.6-20.1$ & 407 & 7.2 & $6.5-7.9$
\end{tabular}

Por síntomas, rinitis

\begin{tabular}{|c|c|c|c|c|c|c|}
\hline Síntomas nasales sin gripa ${ }^{\ddagger}$ & 219 & 43.6 & $40.0-48.0$ & 1553 & 28 & $26.3-29.0$ \\
\hline Síntomas nasales más oculares ${ }^{\ddagger}$ & 109 & 22.0 & $18.2-26.0$ & 907 & 16.1 & 15.1-17.1 \\
\hline
\end{tabular}

Por síntomas, eczema

\begin{tabular}{lcccccc} 
Ronchas alguna vez $^{*}$ & 89 & 18.0 & $15.0-21.4$ & 706 & 13.0 & $12.0-13.4$ \\
\hline Ronchas últimos 12 meses $^{\ddagger}$ & 58 & 12.0 & $8.9-15.0$ & 467 & 8.2 & $7.5-9.0$
\end{tabular}

* Prevalencia acumulada

$\ddagger$ Prevalencia actual

$\mp p<0.001$ calculada con la prueba de $\chi^{2}$ de Pearson

$\mathrm{n}=$ número de niños 


\section{Cuadro V \\ Prevalencia de asma, rinitis y eCzema por tipo de escuela. Ciudad Juárez, Chinuahua, México, 1998-1999}

\begin{tabular}{|c|c|c|c|c|c|c|}
\hline \multirow[b]{2}{*}{ Variable } & \multicolumn{3}{|c|}{ Escuela privada } & \multicolumn{3}{|c|}{ Escuela pública } \\
\hline & $n$ & $\%$ & IC95\% & $n$ & $\%$ & IC95\% \\
\hline \multicolumn{7}{|l|}{ Por diagnóstico médico } \\
\hline Asma* & 30 & 8.0 & $5.5-11.3$ & 234 & 4.1 & $3.6-4.6$ \\
\hline Bronquitis asmática* & 21 & 5.6 & $3.5-8.5$ & 281 & 4.9 & $4.3-5.5$ \\
\hline Asma y bronquitis asmática* & 40 & 10.6 & $7.7-4.1$ & 379 & 6.5 & $5.9-7.2$ \\
\hline Rinitis* & 39 & 10.4 & $7.5-14.0$ & 69 & 4.7 & $4.1-5.3$ \\
\hline Eczema* & 33 & 8.8 & $6.1-12.1$ & 265 & 4.6 & $4.1-5.2$ \\
\hline
\end{tabular}

Por síntomas, asma

\begin{tabular}{|c|c|c|c|c|c|c|}
\hline Tos nocturna ${ }^{\ddagger}$ & 45 & 11.9 & $8.8-15.6$ & 1122 & 19.4 & $18.4-20.4$ \\
\hline Tos persistente ${ }^{\ddagger}$ & 63 & 17.0 & $13.1-21.0$ & 1568 & 27.1 & $26.0-28.2$ \\
\hline Sibilancia alguna vez* & 94 & 25.1 & $21.0-30$ & 1177 & 20.4 & $19.3-21.4$ \\
\hline$\overline{\text { Sibilancia actual }}{ }^{\ddagger}$ & 30 & 8.0 & $5.5-11.2$ & 461 & 8.0 & $5.5-11.3$ \\
\hline
\end{tabular}

Por síntomas, rinitis

\begin{tabular}{lrrrrrr} 
Síntomas nasales sin gripa $^{\ddagger}$ & 139 & 37.1 & $32.2-42.2$ & 1633 & 28.3 & $27.1-29.5$ \\
\hline Síntomas nasales más oculares $^{\ddagger}$ & 61 & 16.2 & $13.0-20$ & 955 & 16.6 & $16.0-18.0$
\end{tabular}

Por síntomas, eczema

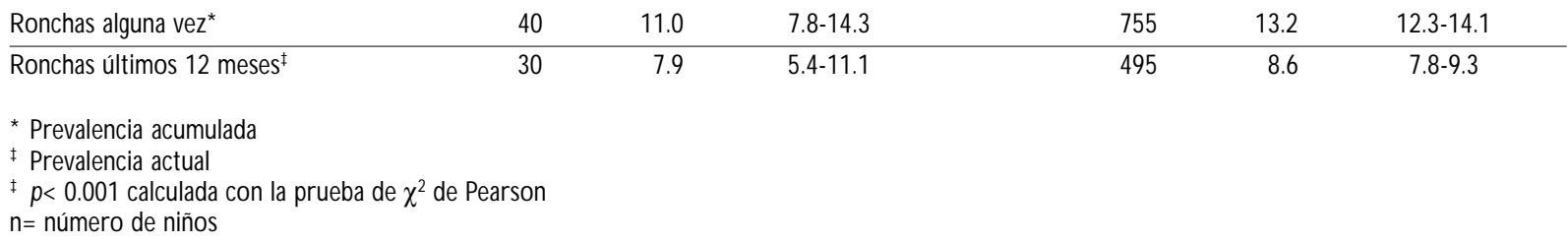

prevalencia encontrada fue de $16.0 \%$ para el estrato 3, siendo igualmente mayor que en los otros dos estratos. Para el caso de rinitis por síntomas, las prevalencias encontradas en los estratos 1 y 2 fueron similares (29.0\% y $29.2 \%$ ). Es importante destacar que no fueron consistentes los hallazgos de mayor riesgo para el estrato 1, considerado como el de mayor contaminación, de acuerdo con las concentraciones de material partículado $\mathrm{PM}_{10}$, por el experto en el tema.

\section{Discusión}

La presente investigación se realizó utilizando la metodología internacional para el estudio del asma y otras enfermedades alérgicas propuesta por el ISAAC, la cual constituye el segundo estudio realizado en México, después del que se hizo en la ciudad de Cuernavaca,
Morelos, ${ }^{11}$ como estudio basal, para detectar las prevalencias y severidad de síntomas del asma, rinitis alérgica y eczema entre la población escolar mediante un cuestionario validado y estandarizado. Según datos proporcionados por el Instituto Mexicano del Seguro Social sobre morbilidad de enfermedades respiratorias, la situación de estos padecimientos ha ido en aumento en el estado de Chihuahua, alcanzando tasas de hasta 22 por 100000 habitantes. Por otro lado, según datos proporcionados por la Secretaría de Salud, las enfermedades respiratorias ocupan el décimo primer lugar dentro de las principales causas de mortalidad en Ciudad Juárez, siendo los más afectados los menores de edad. ${ }^{13}$

Poder evaluar la situación real de estos padecimientos en Ciudad Juárez, nos permitió hacer una comparación, en tiempo y espacio, con estudios similares 
en otras ciudades, lo que servirá de base para futuras investigaciones en el país y en el mundo.

En los estudios epidemiológicos sobre asma en población infantil una estrategia muy sencilla y ampliamente utilizada es el uso del cuestionario como instrumento de trabajo; previo a la utilización del cuestionario estandarizado por el ISAAC era común utilizar otro tipo de instrumento, como el desarrollado por la American Thoracic Society (ATS), el cual incluía preguntas sobre los principales síntomas y enfermedades respiratorias, pero no cubría extensivamente los síntomas de los otros padecimientos estudiados. Una de las bondades de la metodología utilizada consiste en incluir todas las definiciones posibles, lo que permite clasificar a un paciente como caso, independientemente de las diferencias que existen en los criterios médicos predominantes, hecho que posibilita de gran manera la comparabilidad de los resultados.

Con la población escolar de la misma edad, el muestreo se seleccionó de manera probabilística, lo que permite inferir los resultados en el resto de la población infantil escolar de Ciudad Juárez, Chihuahua.

La prevalencia de asma bajo la definición de diagnóstico médico de asma en escolares de Ciudad Juárez, Chihuahua, fue de 6.7 para los niños de primer grado ( 6 a 8 años), y de 7.4 para el grupo de sexto año (11 a 14 años), la cual es con esta misma metodología ligeramente más elevada que las prevalencias encontradas en el estudio realizado en Cuernavaca, Morelos, ${ }^{11}$ cuya prevalencia de asma por diagnóstico médico fue de 5.7 (6 a 8 años) y de $5.9 \%$ (11 a 14 años).

Preguntar sobre la presencia de síntomas de los padecimientos brinda otro acercamiento, quizá más real, a la prevalencia. El estudio realizado en Cuernavaca, Morelos, México, ${ }^{11}$ y en las ciudades de Campos Gerais y Minas Gerais, Brasil ${ }^{20}$ estiman una prevalencia para sibiliancia actual de 8.9 y $5.8 \%$ para los niños de 6 a 8 , y para los niños de 7 a 8 años, respectivamente, y de 6.6 y $6 \%$ para los niños de 11 a 14 años y de 13 a 14 años, frente a los 9.7 y $5.8 \%$ encontrado en este trabajo.

Entre otras investigaciones que reportan síntomas, se pueden cotejar los datos aquí obtenidos con el estudio comparativo hecho por Habbick BF y colaboradores ${ }^{21}$ en el que se utilizó también la metodología del ISAAC en Hamilton y Saskatoon, Canadá, donde la prevalencia de sibilancia actual entre los niños de 6 a 7 años, y de los niños de 13 a 14 años fue de 20.1 y $30.6 \%$ en Hamilton, y de 14.1 y $24.0 \%$ en Saskatoon, respectivamente, frente a 9.7 y $5.8 \%$ de nuestros grupos de edad estudiados.

En las ciudades de Almería, Barcelona, Bilbao, Bahía de Cádiz, Cartagena, Castellón, Pamplona, Va- lencia y Valladolid, de España, ${ }^{22}$ utilizando igualmente la metodología del ISAAC, se reportan prevalencias de sibilancia en los últimos 12 meses de $15.4 \%$ en hombres contra $12.6 \%$ en mujeres para el grupo de edad de 13 a 14 años; el análisis mostró que para los niños de esos mismos grupos de edad las prevalencias encontradas fueron bajas para Valladolid, Almería, Castellón y Pamplona, sin embargo, resultaron altas en comparación con nuestros datos.

Asimismo, los hallazgos en las ciudades de Melbourne, Sydney, Adelaide y Perth, en Australia, ${ }^{23}$ se encuentran igualmente por arriba de las prevalencias encontradas en esta investigación.

En este estudio los resultados por sibilancia actual fueron mayores respecto al efectuado en Inglaterra, ${ }^{24}$ para niños de 12 a 14 años, en el cual la prevalencia actual de sibilancia fue de $5 \%$.

Es posible hacer la comparación con estos datos, por primera vez, gracias al uso de esta metodología. Con otros estudios realizados en América Latina con diferente metodología ${ }^{8-10,15}$ y con diferentes definiciones operativas esta comparación no es posible.

Bajo la definición de diagnóstico médico, y al comparar los datos de la prevalencia de asma con la de los estudios de otros países en donde se utilizó la misma metodología, se debe tener presente que en cada uno de estos países la práctica médica es distinta, lo que hace diferente la definición de diagnóstico médico. Por otro lado, se debe considerar igualmente que no toda la población tiene acceso a los servicios de salud, por lo que el valor real de la prevalencia de asma en la estudiada, con base en la definición de diagnóstico médico, puede estar subestimado. A pesar de esta limitación es claro que la prevalencia por diagnóstico médico es muy importante porque puede ser un indicador de asma severa.

Cabe tener en cuenta que ésta es sólo una de las definiciones operacionales que ofrece este instrumento, y que es obvia la ventaja de poder comparar síntomas, tanto en frecuencia como en severidad, sin limitarse exclusivamente a esta definición, como ocurría con metodologías anteriores.

A este respecto, vale la pena mencionar que, si bien la unificación de la definición operacional, para efectos de investigación y evaluación es muy importante, no lo es menos para efectos clínicos por las implicaciones que tiene el diagnóstico en costos y en manejo del paciente, ya que el asma es una de las principales causas de admisión en los servicios de pediatría de los hospitales. ${ }^{24}$

La mayor prevalencia de síntomas de asma y eczema se encontró en el estrato 3 , que corresponde a la zona periférica de la ciudad y a la zona considerada 
como medianamente contaminada; ello se consiguió tomando como criterio de estratificación las concentraciones de material particulado $\mathrm{PM}_{10}$, aunque es importante mencionar que las diferencias encontradas entre las concentraciones de este contaminante para cada uno de los estratos son mínimas, ya que las características geográficas de la ciudad permiten que los contaminantes tengan un patrón de dispersión semejante. Con el análisis actual no se puede concluir que en esta zona los síntomas sean más prevalentes por una contaminacion ambiental, puesto que en esta región se encuentra ubicada una gran parte de la población con bajos recursos económicos, lo que puede estar haciendo confusa esta relación, además, como ya se estableció, la prevalencia de síntomas es mayor en las escuelas públicas, lo cual puede asociarse con el nivel socioeconómico, no obstante, es necesario estudiar con más detalle las características sociodemográficas y los factores de riesgo ambientales.

Se puede afirmar que la prevalencia de asma, rinitis y eczema fue significativamente mayor en los niños con antecedentes familiares de asma y que, por nivel socioeconómico, se observó una mayor prevalencia de diagnóstico médico entre los niños que asistían a escuelas privadas, en tanto que la prevalencia de síntomas de estos tres padecimientos fue mayor entre los niños que asisten a escuelas públicas, esto es, los que tienen un nivel socioeconómico más bajo.

En cuanto a las limitaciones del estudio, es importante considerar la posibilidad de sesgos en el supuesto de que las personas con hijos que padecen alguno de los problemas estudiados tendieran a contestar más que las otras personas o a aceptar en mayor medida la inclusión en el estudio. Para evitar esto se hizo una caracterización de pérdidas mediante la aplicación de un cuestionario más corto a los niños que no respondieron en la primera etapa, sin haber encontrado diferencias significativas entre los padecimientos estudiados. La tasa de respuesta fue de $92 \%$ lo que rebasa por mucho las tasas de respuesta reportadas en otros estudios ejecutados con metodologías similares. ${ }^{25,26}$ Por otra parte, la tasa de respuesta tan alta disminuye considerablemente la posibilidad de un sesgo de este tipo.

Debido a que en la metodología del estudio se utilizó un cuestionario, en este caso autoadministrado, es posible tener mala clasificación de los sujetos ya que la respuesta depende, por un lado, de la memoria del sujeto que responde a los cuestionamientos, y por otro depende de la interpretación que le dé a la pregunta. Sin embargo, para clasificar a un sujeto como asmático no se tomó únicamente una pregunta, sino que se insertaron a lo largo del cuestionario preguntas for- muladas de diferente manera, relacionadas con diversos síntomas que en conjunto establecieron la definición de los padecimientos, lo que aumenta la validez del estudio. No se puede descartar, sin embargo, la presencia de un error aleatorio que, en todo caso, estaría sesgando el estimador hacia el valor nulo.

Para verificar la reproducibilidad del instrumento se practicó una prueba en una submuestra de 85 escolares, a partir de la cual se obtuvieron valores satisfactorios (Kappa entre 0.67 y 1.00) para las preguntas evaluadas. ${ }^{27}$

Otro punto importante fue que el llenado de los cuestionarios para los dos grupos estudiados, lo hicieron los padres de familia, lo que brinda una mayor posibilidad de concordancia en las respuestas, situación que no se observa en otros estudios, ya que para el grupo de mayor edad el cuestionario lo respondieron los niños.

Finalmente, este estudio proporciona datos sobre prevalencias de enfermedades alérgicas en niños, que se podrían monitorear en el futuro para determinar tendencias en el tiempo. Contribuye, además, a la validación y a la adaptación del método en la población estudiada, lo que puede permitir posibles intervenciones.

\section{Referencias}

1. Taylor W R, N ewacheck PW. Impact of childhood asthma on health. Pediatrics 1992;90:657-662.

2. Yunginger JW, Reed $C E, O$ 'C onnell EJ, Melton LJ, 0 'Fallon W M, Silverstein MD.A community-based study of the epidemiology of asthma. I. Incidence rates, 1964-83.Am Rev Respir D is 1992;146:888-894.

3.The International Study of Asthma and Allergies in Childhood (ISAAC) Steering Committee. Worldwide variation in prevalence of symptoms of asthma, allergic rhinoconjunctivitis, and atopic eczema:ISAAC. Lancet 1998 A pr 25;351(9111):1225-1232.

4. Bernhisel BJY. Sampson HA. Hipersensibilidad a los alimentos y dermatitis atópica. En: Fireman P, ed. Clínicas Pediátricas de N orteamérica 1988. México, D.F.: N ueva Editorial Interamericana, 1989:1209-1226.

5.Taylor W R, N ewacheck PW. Impact of childhood asthma on health. Pediatrics 1992;90(5):657-662.

6. Ehrlich RI, Toit DD, Jordan E, Yamik JA, W einberg EG, Zwarenstein M. Prevalence and reliability of asthma symptoms in primary school children in Cape Town. Int J Epidemiol 1995;24:1138-1146.

7. Pedroza A, Velázquez R, Huerta J, G utiérrez G, G arcía R. Frequency of asthma. Study in Mexican children. Proceedings of the XIV International Congress of Allergology and Clinical Inmunology; 19910 ct 13-18; Kioto, Japón.

8. Canseco C, Leal L, Mora H, Galindo G. Epidemiología de las enfermedades alérgicas en el área metropolitana de Monterrey. Rev Alerg Mex 1991;38 (3):95-101.

9. González JG, Becerra LE, Arévalo MA. Prevalencia del asma bronquial en población escolar en la ciudad de Guadalajara, Jal. México. Rev Alerg Mex 1992;39(1):3-7. 
10.Vargas MH, Sienra JJ, Díaz-M ejía GS, O Ivera-C astillo R, León-G onzález MD.Aspectos epidemiológicos del asma infantil en México. Gac Med Mex 1994;132:255-265

11.Tatto-C ano MI, Sanín-A guirre LH, GonzálezV, Ruiz-Velasco S, Romieu I. Prevalencia del asma, rinitis y eczema en niños escolares de Cuernavaca, Morelos. Salud Publica Mex 1997;39(6):497-506.

12. Moyes CD,W aldon J, Romadas D,C rane J, Pearce N . Respiratory symptoms and environmental factors in school children in the Bay of Plenty. N Z. Med J 1995;8 (108):358-361.

13. Instituto $N$ acional de Estadística G eografía e Informática. XI Censo de Población yVivienda 1990. C uaderno Estadístico Municipal, C iudad Juárez Chihuahua. Aguascalientes, A guascalientes: IN EG ; 1990.

14. Steen-Jonhsen J, Bolle R Holt J, Benan K, Magnus P. Impact of pollution and place of residence on atopia diseases among schoolchildren in Telemark; C ounty, N orway. Pediatr Allergy Immunol 1995;6(4):192-199.

15. Salas-Ramírez M, Segura-Méndez N H, Martínez-Cairo CS. Tendencia de la mortalidad por asma en México. Bol 0 ficina Sanit Panam 1994;116 (4): 298-306.

16.W hite MC, Etzel RA,W ilcox W D, Lloyd C. Exacerbations of childhood asthma and ozone pollution en Atlanta. Environ Res 1994; 65(1): 56-68.

17. Dekker C, Dales R, Bartlett S, Brunekreef B, Zwanenburg H. Childhood asthma and indoor environment. Chest 1991;100(4):922-926.

18. Schwartz J, G old D, D ocker D W, W eiss ST, Speizer FE. Predictors of asthma and persistent wheeze in a national sample of children in the U nited States.Am Rev Respir Dis 1990;142:555-562.

19. International Study of Asthma and Allergies in Childhood (ISAAC). Manual for ISAAC .Averland (N 2) Bocuhm (FRG):ISSAC Cordinating Committee, 1992.

20. C amargos PA, C astro RM, Feldman JS. Prevalence of symptoms related to asthma in school children of Campos Gerais, Brazil. Rev Panam Salud Publica 1999 Jul;6(1):8-15.
21. Habbick BF, Pizzichini MM, Taylor B, Rennie D, Senthilselvan A, Sears MR. Prevalence of asthma, rhinitis and eczema among children in $2 \mathrm{C}$ anadian cities:The International Study of Asthma and Allergies in Childhood. CMAJ 1999 Jun 29;160(13):1824-1828.

22. A guinaga-0 ntoso I,Arnedo-PenaA, Bellido J, G uillén-G rima F, SuárezVarela MM.The prevalence of asthma-related symptoms in 13-14-year-old children from 9 Spanish populations. The Spanish Group of the ISAAC Study (International Study of Asthma and Allergies in Childhood). Med Clin (Barc) 1999 Feb 13;112(5):171-175.

23. Robertson CF, D alton MF, Peat JK, Haby MM, Bauman A, Kennedy JD et al.Asthma and other atopic diseases in A ustralian children.Australian arm of the International Study of Asthma and Allergy in C hildhood. Med J A ust 1998; May 4;168(9):434-438.

24. Burr ML,Anderson HR,Austin JB, Harkins LS, Kaur B, Strachan DP et al. Respiratory symptoms and home environment in children:A national survey.Thorax 1999;54(1):27-32.

25. Sole D,Yamada E,VanaAT, C osta-C arvalho BT, N aspitz CK. Prevalence of asthma and related symptoms in school-age children in Sao Paulo, Brazil- International Study of Asthma and Allergies in Children (ISAAC). J Asthma 1999;36(2):205-212.

26. Montefort S, Lenicker HM, C aruna S, A gius Muscat H.Asthma, rhinitis and eczema in Maltese 13-15 year-old schoolchildren - Prevalence, severity and associated factors. International Study of Asthma and Allergies in Childhood (ISAAC). Clin Exp Allergy 1998;28(9):1089-1099.

27. Silva-Ayfaguer L. Excursión a la regresión logística. La Habana, Cuba: Díaz de Santos, 1994:101-102. 